\title{
Abdominal Burkitt's lymphoma with renal involvement
}

\author{
H Moodley, ${ }^{1}$ MB ChB; N Mahomed, ${ }^{1,2}$ MB BCh, FCRad; S Andronikou, ${ }^{1}$ MB BCh, FCRad, FRCR, PhD \\ ${ }^{1}$ Radiology Department, Faculty of Health Sciences, University of the Witwatersrand, Johannesburg, South Africa \\ ${ }^{2}$ Department of Paediatric Radiology, Chris Hani Baragwanath Hospital, Johannesburg, South Africa
}

Corresponding author: $N$ Mahomed (nasreen.mahomed@wits.ac.za)

\begin{abstract}
Endemic Burkitt's lymphoma is the most frequent childhood cancer in Africa, commonly presenting with involvement of the abdominal viscera. Imaging plays a critical role in suggesting the diagnosis to expedite treatment initiation, as the tumour has a rapid doubling time of less than 24 hours. This case report illustrates the classic nodal and infrequent renal appearance on computed tomography scans.
\end{abstract}

S Afr J CH 2013;7(2):79. DOI:10.7196/SAJCH.548

Burkitt's lymphoma is one of the most common types of non-Hodgkin's lymphoma in childhood. ${ }^{[1]}$ In Africa, it is the most frequent cancer of childhood. ${ }^{[2]}$ The World Health Organization classification stratifies Burkitt's lymphoma into endemic, sporadic and HIV-associated variants. ${ }^{[2]}$ The endemic subtype is found in Africa, usually in the 4 - 7-year-old age group and involving extranodal sites, typically the jaw or abdominal viscera. ${ }^{[2]}$ Epstein-Barr virus is the causative agent in almost all cases. ${ }^{[2]}$

\section{Case report}

A 3-year-old girl of unknown HIV status presented with a 1-week history of vomiting and abdominal distension. An urgent abdominal ultrasound scan demonstrated a para-aortic nodal mass and diffuse

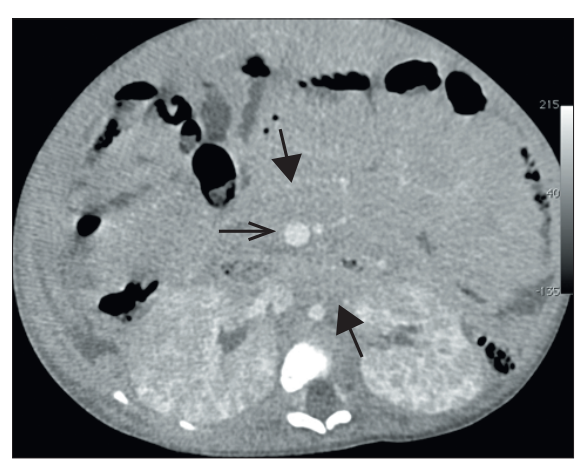

Fig. 1. Contrast-enhanced computed tomography scan of the abdomen demonstrating a lobulated paraaortic nodal mass encasing and elevating the aorta. The 'sandwich sign' refers to encasement on either side of the superior mesenteric artery and superior mesenteric vein (open arrow) by lobulated, confluent mesenteric nodal masses (arrow heads), resembling a sandwich's buns and filling. The 'tigroid' renal enhancement pattern suggests diffuse lymphomatous infiltration. There is associated diffuse infiltration of the bowel. infiltration of the bowel and kidneys. A contrast-enhanced computed tomography (CT) scan of the abdomen confirmed the para-aortic nodal mass with encasement of the aorta, superior mesenteric vein and inferior mesenteric vein ('sandwich sign ${ }^{\text {?[1] }}$ ) (Fig. 1), diffuse infiltration of the bowel and a 'tigroid' enhancement pattern of both kidneys as well as mesenteric metastases (Fig. 1). A significant left pleural effusion was present, but no mediastinal or hilar lymphadenopathy was identified.

Ultrasound-guided renal biopsy demonstrated a high-grade B-cell lymphoma, with morphological and immunophenotypic features consistent with a Burkitt's lymphoma. The patient was initiated on chemotherapy but died of acute tumour lysis syndrome 2 days thereafter.

\section{Discussion}

Endemic Burkitt's lymphoma classically presents with abdominal involvement, commonly bowel, nodal and secondary renal involvement. ${ }^{[1]}$ Gastrointestinal involvement is the most common presentation, with infiltration of the wall of the ileocaecal region, appendix and ascending colon being most frequent. ${ }^{[1]}$ Nodal involvement may result in a confluent mass encasing and displacing the abdominal vasculature, ${ }^{[1]}$ as seen in our patient. Burkitt's lymphoma has the highest incidence of renal involvement of the childhood lymphomas, usually in the terminal stage. ${ }^{[1]}$ The three infiltration patterns documented in order of frequency are: (i) numerous bilateral small nodules; (ii) a mass lesion; and (iii) diffuse infiltrates, as described in our patient. ${ }^{[1]}$

The differential diagnosis is vast, as lymphoma can affect almost any organ. ${ }^{[1]}$
It includes inflammatory, benign and neoplastic conditions. ${ }^{[1]}$ Notably, in Africa tuberculosis must be considered in a patient with a nodal mass. Tuberculosis is distinguished by a para-aortic and portahepatis nodal distribution, with necrotic or calcified lymphadenopathy prior to treatment. ${ }^{[3]}$ Lymphoma more frequently involves pelvic lymph nodes, and nodes only calcify after treatment. ${ }^{[3]}$

\section{Conclusion}

Endemic Burkitt's lymphoma must be considered when a child presents with an abdominal mass in the African environment. It classically presents with abdominal involvement, commonly bowel, nodal and secondary renal involvement. Imaging in the form of ultrasound, CT and magnetic resonance imaging (MRI) is important in suggesting the diagnosis and staging of abdominal disease, and image-guided biopsy is an invaluable tool in making the definitive diagnosis. This expedites treatment initiation, as Burkitt's lymphoma has a rapid tumour doubling time of less than 24 hours. ${ }^{[4]}$

\section{References}

1. Toma P, Granata C, Rossio A, et al. Multimodality imaging of Hodgkin disease and non-Hodgkin lymphoma in children. Radiographics 2007;27(5):1335-1354. [http:// dx.doi.org/10.1148/rg.275065157]

2. Ferry JA. Burkitt's lymphoma: Clinicopathologic features and differential diagnosis. The Oncologist 2006;11(4):375-383. [http://dx.doi.org/10.1634/ theoncologist.11-4-375]

3. Andronikou S, Welman CJ, Kader E. The CT features of abdominal tuberculosis in children. Pediatr Radiol 2002;32(2):75-81.

4. Vade A, Blane CE. Imaging of Burkitt's lymphoma in pediatric patients. Pediatr Radiol 1985;15(2):123-126 